


Kraehmer U, Rovi A. A Lie-Rinehart algebra with no antipode.

Communications in Algebra 2015, 43(10), 4049-4053.

\title{
Copyright:
}

CUlrich Krähmer and Ana Rovi

This is an Open Access article. Non-commercial re-use, distribution, and reproduction in any medium, provided the original work is properly attributed, cited, and is not altered, transformed, or built upon in any way, is permitted.

DOI link to article:

http://dx.doi.org/10.1080/00927872.2014.896375

Date deposited:

$23 / 09 / 2015$

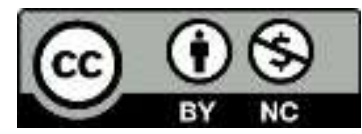

This work is licensed under a Creative Commons Attribution-NonCommercial 3.0 Unported License 


\section{A Lie-Rinehart Algebra with No Antipode}

\section{Ulrich Krähmer \& Ana Rovi}

To cite this article: Ulrich Krähmer \& Ana Rovi (2015) A Lie-Rinehart Algebra with No Antipode, Communications in Algebra, 43:10, 4049-4053, DOI: 10.1080/00927872.2014.896375

To link to this article: http://dx.doi.org/10.1080/00927872.2014.896375

$$
\text { (2) Copyright Ulrich Krähmer and Ana Rovi }
$$

Published online: 06 Jul 2015.

Submit your article to this journal $₫$

LII Article views: 126

Q View related articles $₫$

View Crossmark data $\asymp$ 


\section{A LIE-RINEHART ALGEBRA WITH NO ANTIPODE \\ Ulrich Krähmer and Ana Rovi \\ School of Mathematics and Statistics, University of Glasgow, Glasgow, UK \\ The aim of this note is to communicate a simple example of a Lie-Rinehart algebra whose enveloping algebra is not a Hopf algebroid, neither in the sense of Böhm and Szlachányi, nor in the sense of Lu.}

Key Words: Hopf algebroids; Lie algebroids; Lie-Rinehart algebras.

2010 Mathematics Subject Classification: 16T05; 57T05; 16S30.

\section{INTRODUCTION}

The enveloping algebra of a Lie algebra is a classical example of a Hopf algebra. Hence it is natural to ask whether the enveloping algebra of a Lie algebroid [12] or more generally of a Lie-Rinehart algebra [13] carries the structure of a Hopf algebroid. It turns out that they always are left bialgebroids (introduced under the name $\times_{R}$-bialgebras by Takeuchi [15]), see [16], and in fact left Hopf algebroids (introduced under the name $\times_{R}$-Hopf algebras by Schauenburg [14]), see [6, Example 2]; see also [5, 11].

However, there is also the definition of a Hopf algebroid due to $\mathrm{Lu}$ [9] and the one due to Böhm and Szlachányi [2] (the latter will be called full Hopf algebroids from now on), which both assume the existence of an antipode satisfying certain axioms. The aim of the present paper is to communicate a concrete example of a Lie-Rinehart algebra whose universal enveloping algebra is not a Hopf algebroid in either of these two settings.

This clarifies further the relation between the three concepts: it is well-known and easily seen that every full Hopf algebroid is a left Hopf algebroid, see [2]. In [1], an example of a full Hopf algebroid was given that is not a Hopf algebroid in the sense of [9], but to the best of our knowledge, it is not known whether every Hopf algebroid in the sense of Lu satisfies the axioms of a full or at least of a left Hopf algebroid, and whether every left Hopf algebroid admits an antipode satisfying

CUlrich Krähmer and Ana Rovi

This is an Open Access article. Non-commercial re-use, distribution, and reproduction in any medium, provided the original work is properly attributed, cited, and is not altered, transformed, or built upon in any way, is permitted. The moral rights of the named author(s) have been asserted.

Received September 23, 2013; Revised February 11, 2014. Communicated by T. Lenagan.

Address correspondence to Ulrich Krähmer, School of Mathematics and Statistics, University of Glasgow, Glasgow G12 8QW, UK; E-mail: ulrich.kraehmer@glasgow.ac.uk 
either of the definitions of [2,9] (a counterexample announced in [8, Remark 3.12] did not appear in print).

In the light of [8, Proposition 3.11], it is known that the enveloping algebras of Lie algebroids [3] and of the Lie-Rinehart algebras associated to Poisson algebras [4, Section (3.2)] are full Hopf algebroids. However, here we prove the following theorem.

Theorem 1.1. Let $K$ be a field, $R:=K[x, y] /\left\langle x \cdot y, x^{2}, y^{2}\right\rangle, L$ be the 1-dimensional Lie algebra with basis $\{\alpha\}$, and $E \in \operatorname{Der}_{K}(R)$ be the derivation with $E(x)=y, E(y)=0$.

1. There is a Lie-Rinehart algebra structure on $(R, L)$ with $R$-module structure on $L$ given by $x \cdot \alpha=y \cdot \alpha=0$ and anchor map given by $\rho(\alpha)=E$.

2. There is no right $V(R, L)$-module structure on $R$ that extends multiplication in $R$.

3. $V(R, L)$ is neither a full Hopf algebroid, nor a Hopf algebroid in the sense of [9].

The note is structured as follows. In Section 2, we recall some basic definitions and prove the implication 2. $\Rightarrow 3$. of Theorem 1.1. In Section 3, we provide a construction method of Lie-Rinehart algebras whose enveloping algebras satisfy part 2, for which the Lie-Rinehart algebra from 1 is a basic example.

\section{BACKGROUND}

This section contains background on Lie-Rinehart algebras [13], see also [4, 7, $8,11]$ for more information. For the corresponding differential geometric notion of a Lie algebroid see [12] and for example [10] for further details.

We fix a field $K$. An unadorned $\otimes$ denotes the tensor product of $K$-vector spaces.

Definition 2.1. A Lie-Rinehart algebra consists of the following elements:

1. A commutative $K$-algebra $(R, \cdot)$;

2. A Lie algebra $\left(L,[-,-]_{L}\right)$ over $K$;

3. A left $R$-module structure $R \otimes L \rightarrow L, r \otimes \xi \mapsto r \cdot \xi, r \in R, \xi \in L$; and

4. An $R$-linear Lie algebra homomorphism $\rho: L \rightarrow \operatorname{Der}_{K}(R)$ satisfying

$$
[\xi, r \cdot \zeta]_{L}=r \cdot[\xi, \zeta]_{L}+\rho(\xi)(r) \cdot \zeta, \quad r \in R, \xi, \zeta \in L
$$

The map $\rho$ is referred to as the anchor map.

There are two fundamental examples: if $R$ is any commutative algebra, one can take $L$ to be $\operatorname{Der}_{K}(R)$ with its usual Lie algebra and $R$-module structure, and $\rho=\mathrm{id}$. The other extreme is $R=K$ and $\rho=0, L$ being any Lie algebra.

In his paper [13], Rinehart generalised the construction of the universal enveloping algebra of a Lie algebra to Lie-Rinehart algebras, see Section 2 therein for the precise construction. The result is an associative $K$-algebra $V(R, L)$ that is generated by the (sum of the) images of a $K$-algebra map

$$
R \longrightarrow V(R, L)
$$


and a Lie algebra map

$$
\left(L,[-,-]_{L}\right) \longrightarrow(V(R, L),[-,-]), \quad \xi \longmapsto \bar{\xi},
$$

where $[-,-]$ denotes the commutator in $V(R, L)$. As Rinehart, we do not distinguish between an element in $R$ and its image in $V(R, L)$ which is justified as the first map is always injective. The construction is such that in $V(R, L)$ one has for all $r \in R, \xi \in L$

$$
[\bar{\xi}, r]=\rho(\xi)(r), \quad r \bar{\xi}=\overline{r \cdot \xi},
$$

where the product in $V(R, L)$ is denoted by concatenation.

As indicated in the introduction, $V(R, L)$ has the structure of a left Hopf algebroid. Its counit endows $R$ with the structure of a left $V(R, L)$-module, in such a way that the induced action of $r \in R$ is given by multiplication, and the induced action of $\xi \in L$ is given by the anchor map. The following fact is well known and yields the implication 2 . $\Rightarrow 3$. in Theorem 1.1.

Lemma 2.2. If $H$ is either a full Hopf algebroid or a Hopf algebroid in the sense of Lu, with antipode $S: H \rightarrow H$, left counit $\varepsilon: H \rightarrow R$, and source and target maps $s, t: R \rightarrow H$, then defining for $h \in H, r \in R$

$$
r h:=\varepsilon(S(h) s(r))
$$

yields a right $H$-module structure on $R$ for which the underlying left $R$-action on $R$ is given by left multiplication.

Proof. The canonical left action of a left bialgebroid $H$ on the base algebra $R$ is given by $h r:=\varepsilon(h s(r))=\varepsilon(h t(r))$, and the antipode of a Hopf algebroid is an algebra antihomomorphism (see [1, Proposition 4.4] respectively [9, Definition 4.1] for the two different notions). Hence (2.3) defines a right action of $H$ on $R$. Finally, one has $S \circ t=s$ (see [1, Definition 4.1 (iii)] respectively [9, Definition 4.1.2.]), so $r t(q)=\varepsilon(S(t(q)) s(r))=\varepsilon(s(q) s(r))=q r$ for all $q, r \in R$.

\section{A LIE-RINEHART ALGEBRA WITHOUT FLAT RIGHT CONNECTION ON $R$}

We now prove Theorem $1.1,1$. and 2 . We begin by considering more generally Lie-Rinehart algebras $(R, L)$ whose $R$-module structure on $L$ is given by a character $\chi: R \rightarrow K$.

Lemma 3.1. Let $(R, \cdot)$ be a commutative $K$-algebra, $\left(L,[-,-]_{L}\right)$ be a Lie algebra, and $\rho: L \rightarrow \operatorname{Der}_{K}(R)$ be a Lie algebra map. Define an $R$-module structure on $L$ by $r \cdot \xi:=\chi(r) \xi$, where $\chi: R \rightarrow K$ is a character on $R$. Then $(R, L)$ is a Lie-Rinehart algebra if and only if $\rho$ is $R$-linear and $\rho(\xi)(r) \in \operatorname{ker} \chi$ for all $r \in R, \xi \in L$.

Proof. This follows as the Leibniz rule (2.1) takes the form

$$
[\xi, \chi(r) \zeta]_{L}=\chi(r)[\xi, \zeta]_{L}+\chi(\rho(\xi)(r)) \zeta
$$

and hence by the $K$-linearity of the bracket becomes equivalent to $\rho(\xi)(r) \in \operatorname{ker} \chi$. 
Note that for these examples, $[-.-]_{L}$ is even $R$-linear, so $L$ is a Lie algebra over $R$. However, in general we have $\rho \neq 0$.

Assume now that $(R, L)$ is a Lie-Rinehart algebra as in the above lemma, and that multiplication in $R$ can be extended to a right $V(R, L)$-module structure on $R$. Denote by $\partial(\xi) \in R$ the element obtained by acting with $\xi \in L$ on $1 \in R$ under this right action. This defines a $K$-linear map $\partial: L \rightarrow R$, and in $V(R, L)$ we have

$$
\rho(\xi)(r)=[\bar{\xi}, r]=\bar{\xi} r-r \bar{\xi}=\bar{\xi} r-\overline{r \cdot \xi}=\bar{\xi} r-\chi(r) \bar{\xi},
$$

so by acting with this element on $1 \in R$, one sees that this map $\partial$ satisfies

$$
\rho(\xi)(r)=\partial(\xi) \cdot(r-\chi(r)) .
$$

A $K$-linear map $\partial$ with this property defines a right $V(R, L)$-module structure extending multiplication on $R$ if and only if it satisfies the condition $\partial\left([\xi, \zeta]_{L}\right)=$ $\rho(\xi)(\partial(\zeta))-\rho(\zeta)(\partial(\xi))$. It also corresponds to a generator of the Gerstenhaber bracket on $\Lambda_{R} L$, see [4], but we shall not need these facts.

Proof of Theorem 1.1, 1. and 2. The first part is verified by explicit computation; the Lie-Rinehart algebra is of the form as in Lemma 3.1 with $\chi$ given by $\chi(x)=\chi(y)=0$.

For 2., take $r=x$ and $\xi=\alpha$ in (3.1). One obtains $y=E(x)=\rho(\alpha)(x)=\partial(\alpha) \cdot x$. However, there is no element $z \in R$ such that $y=z \cdot x$.

Carrying out Rinehart's construction explicitly yields a presentation of the associative $K$-algebra $V(R, L)$ in terms of generators $x, y, \bar{\alpha}$ satisfying the relations

$$
\bar{\alpha} x=y, \quad \bar{\alpha} y=x \bar{\alpha}=y \bar{\alpha}=x^{2}=y^{2}=x y=y x=0 .
$$

Hence $V(R, L)$ has a $K$-linear basis given by $\left\{\bar{\alpha}^{n}, x, y\right\}_{n \in \mathbb{N}}$. The source and target maps are both the inclusion of $R$ into $V(R, L)$. Hence one can also see directly that $V(R, L)$ admits no antipode: $S$ would satisfy $S(x)=x, S(y)=y$ and one would have $y=S(y)=S(\bar{\alpha} x)=S(x) S(\bar{\alpha})=x S(\bar{\alpha})$, but there is no element $z \in V(R, L)$ such that $y=x z$.

\section{ACKNOWLEDGMENT}

A.R. thanks José Figueroa O'Farrill for his encouragement, and Gwyn Bellamy for remarks about differential operators.

\section{FUNDING}

U.K. acknowledges support by the EPSRC grant (No. EP/J012718/1) "Hopf algebroids and Operads" and the Polish Government Grants 2012/06/M/ST1/00169; A.R. is funded by an EPSRC DTA grant.

\section{REFERENCES}

[1] Böhm, G. (2009). Hopf Algebroids. Handbook of Algebra, Vol. 6. Amsterdam: Elsevier/North-Holland, pp. 173-235. 
[2] Böhm, G., Szlachányi, K. (2004). Hopf algebroids with bijective antipodes: Axioms, integrals, and duals. J. Algebra 274(2):708-750.

[3] Evens, S., Lu, J.-H., Weinstein, A. (1999). Transverse measures, the modular class and a cohomology pairing for Lie algebroids. Quart. J. Math. Oxford Ser. (2) 50(200): $417-436$.

[4] Huebschmann, J. (1998). Lie-Rinehart algebras, Gerstenhaber algebras and Batalin-Vilkovisky algebras. Ann. Inst. Fourier (Grenoble) 48(2):425-440.

[5] Huebschmann, J. (2008). The Universal Hopf Algebra Associated with a HopfLie-Rinehart Algebra. Preprint arXiv:0802.3836.

[6] Kowalzig, N., Krähmer, U. (2010). Duality and products in algebraic (co)homology theories. J. Algebra 323(7):2063-2081.

[7] Kowalzig, N. (2009). Hopf algebroids and their cyclic theory. Ph.D. thesis, Universiteit Utrecht.

[8] Kowalzig, N., Posthuma, H. (2011). The cyclic theory of Hopf algebroids. J. Noncommut. Geom. 5(3):423-476.

[9] Lu, J.-H. (1996). Hopf algebroids and quantum groupoids. Internat. J. Math. 7(1): 47-70.

[10] Mackenzie, K. (1987). Lie groupoids and Lie algebroids in differential geometry. London Mathematical Society Lecture Note Series, Vol. 124. Cambridge: Cambridge University Press.

[11] Moerdijk, I., Mrčun, J. (2010). On the universal enveloping algebra of a Lie algebroid. Proc. Amer. Math. Soc. 138(9):3135-3145.

[12] Pradines, J. (1967). Théorie de Lie pour les groupoïdes différentiables. Calcul différentiel dans la catégorie des groupoïdes infinitésimaux. C. R. Acad. Sci. Paris Sér. $A-B, 264: \mathrm{A} 245-\mathrm{A} 248$.

[13] Rinehart, G. S. (1963). Differential forms on general commutative algebras. Trans. Amer. Math. Soc. 108:195-222.

[14] Schauenburg, P. (2000). Duals and doubles of quantum groupoids ( $\times_{R}$-Hopf algebras). New trends in Hopf algebra theory (La Falda), Contemp. Math. 267:273-299. Providence, RI: Amer. Math. Soc., 2000.

[15] Takeuchi, M. (1977). Groups of algebras over $A \otimes \bar{A}$. J. Math. Soc. Japan 29(3): 459-492.

[16] Xu, P. (2001). Quantum groupoids. Comm. Math. Phys. 216(3):539-581. 